$\overline{\text { Note }}$

\title{
Evaluation of a Novel Rapid Detection System for Yeasts and Molds
}

\author{
KEI TOMINAGA*, TOSHIHIRO II, FUMI KASHIWABARA, RYOTARO HARA, \\ YOSHIHIKO UESAKA, AND HIDEMASA KODAKA
}

\author{
Research Department, Nissui Pharmaceutical Co. Ltd., \\ 1075-2 Hokunanmoro, Yuki, Ibaraki 307-0036, Japan
}

Received 17 May, 2010/Accepted 26 July, 2010

\begin{abstract}
A novel system, the NISSUI rapid detection system, has been developed to rapidly detect yeasts and molds in food. This system consists of a liquid medium containing resazurin as a redox indicator, a unique original micro-well dish containing 676 micro-wells, and a fluorescence-monitoring instrument with an incubator. To evaluate this system, orange juice, milk, and physiological saline solutions artificially inoculated with yeasts or molds were used as samples. Comparison of the new system used at $28^{\circ} \mathrm{C}$ for $48 \mathrm{~h}$ with a spread-plate method using a potato-dextrose-agar plate at $25^{\circ} \mathrm{C}$ for $120 \mathrm{~h}$ yielded a correlation coefficient $(r)$ of 0.95 . Our data reveal that the new method considerably shortens the time required for detection of yeasts and molds in food.
\end{abstract}

Key words : Yeast/Mold/Rapid detection/Food/Beverage.

Yeasts and molds are widely distributed throughout the environment, for example, in soil, air, and crops including fruits (Tournas et al., 2006, Tournas and Katsoudas, 2005). They also invade processed food. Most ready-to-drink beverages have high water activity (Aw), which typically allows microbial growth. They are usually characterized by low $\mathrm{pH}$ values, which restrict the growth of microorganisms. However, low pH value allows the growth of specific microbial groups such as yeasts and molds, because of their broad acid requirements (Wyatt et al., 1995). Therefore, the detection and enumeration of yeasts and molds in food and also in ready-to-drink beverages are an integral part of good food-quality assurance. Moreover, the conventional plate method for estimating and detecting yeasts and molds requires incubation at $25^{\circ} \mathrm{C}$ for 5-7 d. Hence, the rapid detection of living microorganisms has become a major challenge in the field of food microbiology (London et al., 2010, Renard et al., 2008).

In an attempt to meet this challenge, we have de-

*Corresponding author. Tel: +81-296-35-1225, Fax: +81296-35-1579, E-mail: k-tominaga (a) yki.nissui-pharm.jp veloped an automated, rapid microbial detection system, the NISSUI rapid detection system, with major emphasis on culturing microorganisms in miniaturized wells. The system is able to detect microorganisms rapidly because the signals derived from microbial metabolic activity are kept in small, restricted volumes. Consequently, signal accumulation occurs immediately. The system is designed to detect yeasts and molds in food rapidly.

The detection of yeasts and molds by the NISSUI rapid detection system was tested by using Aspergillus niger ATCC 16404 (American Type Culture Collection, Manassas, Va), Cladosporium cladosporioides JCM 10680 (Japan collection of microorganism, Wako, Japan), Penicillium glabrum NBRC 5692 (NITE Biological Resource Center, Kisarazu, Japan), Fusarium solani NBRC 5232, Aureobasidium pullulans NBRC 100716, Paecilomyces variotii NBRC 33284, Candida albicans JCM 1542, Saccharomyces cerevisiae ATCC 9080, Pichia anomala JCM 1544, Schizosaccharomyces pombe JCM 1846, Rhodotorula glutinis JCM 8167, and Zygosaccharomyces bailii NBRC 10667.

Mold strains were transferred to potato dextrose 
agar and incubated at $28^{\circ} \mathrm{C}$ for $5-7 \mathrm{~d}$ for production of conidia. The fungal colonies were covered with physiological saline solution containing $0.05 \%$ tween80 , and suspensions were made by gently probing the surface with the tip of a Pasteur pipette, generating a mixture of conidial and hyphae fragments. Each suspension was then filtered with sterile gauze and the number of conidia was counted by a hemocytometer. Yeast strains were transferred to potato dextrose agar and incubated at $28^{\circ} \mathrm{C}$ for $5-7 \mathrm{~d}$. The yeast colonies formed on potato dextrose agar were dissolved in physiological saline solution and counted by a hemocytometer. Prepared yeasts and molds were artificially inoculated into orange juice and milk. The orange juice and milk used in this research were obtained from a local supermarket. A comparative study was done by using physiological saline solutions. The $\mathrm{pH}$ value was adjusted to 5.8 by sterile $1 \mathrm{~N}-\mathrm{NaOH}$ for the microbial detection in orange juice. Orange juice, milk, and physiological saline solutions were artificially inoculated with a single yeast or mold strain prepared as indicated above.

The NISSUI rapid detection system consists of a unique micro-well culture dish, and a fluorescencemonitoring instrument with an incubator and a liquid medium. The unique micro-well culture dish was designed to contain 676 micro wells. The external dimensions of a single, original 676-micro-well dish are $52 \times 52 \mathrm{~mm}$, and the size of each well is $1.2 \times 1.2$ $\times 8.5 \mathrm{~mm}$, with capacity of $13.7 \mu \mathrm{L}$ per well. The $676-$ micro-well dishes were gamma-irradiated for aseptic use and hydrophilically coated. Hydrophilic coating allows for separations of sample solutions into each micro-well without any special circumstance or equipment. Each 676-micro-well dish consisted of a polystyrene plate and a polypropylene lid. The plate and the lid were manufactured by injection molding. The original 676-micro-well dish was manufactured by Sumitomo Bakelite Co. Ltd.

The original instrument for fluorescence monitoring was designed to accommodate 4 original 676-microwell dishes, allowing the incubation of the 676-microwell dishes at a constant temperature and measurement of fluorescence intensity (excitation $540 \mathrm{~nm} /$ emission $590 \mathrm{~nm}$ ) simultaneously within 15 min. Important features of the system include exact positioning for independent measurement of each micro-well, and use of a beam with diameter smaller than the bottom aspect of the micro-well to avoid cross-talk measurements between different wells. The original fluorescence-monitoring instrument was manufactured by Shoko Scientific Co. Ltd.

The liquid media used for the system was potatodextrose broth supplemented with chloramphenicol (Daiichi Sankyo Co. Ltd., Tokyo, Japan) and resazurin (Sigma-Aldrich, St. Louis, Mo). Chloramphenicol was added to inhibit bacterial growth. The metabolic activity of growing microorganisms may reduce resazurin to a fluorogenic substrate, resorufin. The fluorescence intensity of

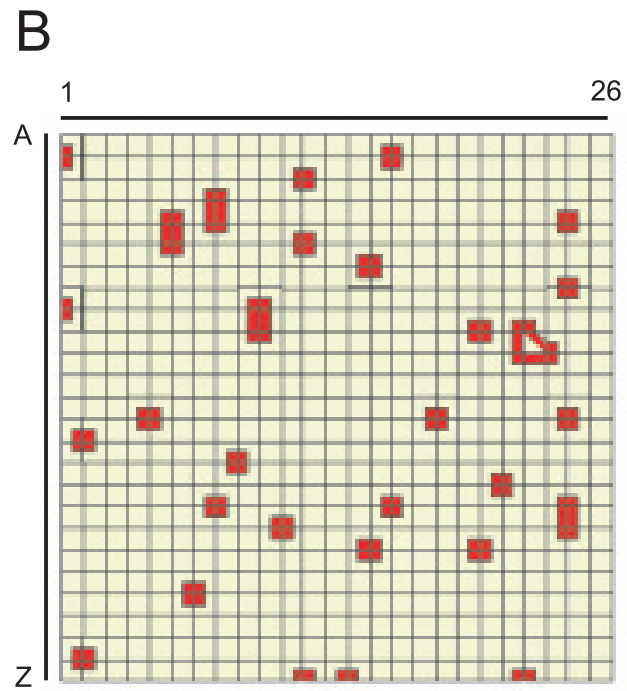

FIG. 1. Incubation of Aspergillus niger ATCC 16404, in the original 676-micro-well dish. (A) The actual image of the cultured dish. Pink and clear wells (color of resorufin and hydro-resorufin) are wells with microorganisms. Micro-wells without microorganisms are blue (color of resazurin). (B) Microorganism-positive wells were determined by the NISSUI rapid detection system. Red spots indicate microorganism-positive wells. 
resorufin was then measured by the original fluorescence-monitoring instrument.

The procedure for yeast and mold detection by this system was initiated by adding $1 \mathrm{ml}$ of a sample solution to $10 \mathrm{ml}$ of potato-dextrose broth supplemented with chloramphenicol and resazurin, followed by gentle mixing. The mixture was then poured into the original 676-micro-well dish and immediately diffused into each micro-well without any special circumstance or equipment. Thereafter, the dishes were loaded into the original fluorescence-monitoring instrument, cultured at $28^{\circ} \mathrm{C}$, and the fluorescence intensity of each micro-well was simultaneously measured every 15 min. Every $15 \mathrm{~min}$, the wells with increased fluorescence intensity were counted, indicating microorganism-positive wells.

The potato-dextrose-agar spread-plate method was carried out with potato-dextrose-agar (Nissui Pharmaceutical Co. Ltd., Tokyo, Japan) supplemented with chloramphenicol. Potato-dextrose-agar was pre-poured and solidified. Afterwards, $0.1 \mathrm{ml}$ sample solutions artificially inoculated with yeasts and molds were aseptically applied onto the potatodextrose-agar by a pipette and the inoculum was spread using a sterile, bacteria spreader. Ten agar plates were used for each sample to obtain the number of microorganisms in a $1 \mathrm{ml}$ sample solution, allowing the comparison with the NISSUI rapid detection system. Plates were incubated at $25^{\circ} \mathrm{C}$ for 5 d. After incubation, colonies were counted.

The original 676-micro-well dishes were tested for the growth of microorganisms (figure 1). The physiological saline solution spiked with Aspergillus niger ATCC 16404 was inoculated into potato-dextrose broth supplemented with chloramphenicol and resazurin. Accordingly, the mixture was poured into the 676-micro-well dishes. As expected, the mixture was immediately separated into each micro-well without any special circumstance or equipment. After 30 $\mathrm{h}$ incubation at $28^{\circ} \mathrm{C}$, the dish was taken out of the fluorescence-monitoring instrument and a picture of the 676-micro-well dish after incubation was taken. The fluorescence intensity data were then analyzed. Comparison of the dish image (figure $1 \mathrm{~A}$ ) with the analyzed fluorescence data (figure 1B) indicated concurrence regarding the positive wells.

Our next trial was to evaluate if the NISSUI rapid detection system could detect yeasts and molds in food. This system and the conventional potatodextrose-agar spread-plate method were compared. Orange juice, milk, and physiological saline solutions artificially inoculated with 12 yeast and mold strains were used as the sample solutions. Orange juice was tested to determine whether the orange pulp would negatively affect the test results. Milk was a good sample to determine whether the intense light absorption will influence the test results. Sample solutions artificially inoculated with yeasts and molds were loaded into the system. Comparisons of the diffusion time of the physiological saline solution, orange juice and milk are presented in Table 1 . The results indicated that orange juice and milk were separated into each micro-well without any difference in diffusion time, despite the fact that orange juice abounds in pulp.

After a sample solution was diffused within the micro-wells, the dish was loaded into the original fluorescence-monitoring instrument. The number of positive wells was counted every $15 \mathrm{~min}$. Results were calculated as log CFU (colony forming unit) of artificially inoculated yeasts and molds per $\mathrm{ml}$ of test sample solutions. Forty-eight hours after incubation, numbers of microorganisms were calculated and plotted against values obtained by the conventional potato-dextrose-agar spread-plate method (figure 2). The correlation coefficient between the NISSUI rapid detection system and conventional potato-dextroseagar spread-plate method was $r(n=36)=0.95$. Linear regression analysis indicated slope and intercept values close to 1.00 and 0.00 , respectively. Furthermore, there was no difference in the correlation coefficient when physiological saline solution, orange juice, or milk was tested. (Table 2). These results suggest a high correlation coefficient between the two methods. A good correlation coefficient was observed between $48 \mathrm{~h}$ determination by the NISSUI rapid detection system and $120 \mathrm{~h}$ incubation by conventional potato-dextrose-agar spread-plate method.

The advantage of this system is that it is easy to use because preparation and sterilization of the media are not required. Counting colonies of yeasts and

TABLE 1. Time required for each solution to diffuse into the original device.

\begin{tabular}{lccc}
\hline \multirow{2}{*}{ solution } & \multirow{2}{*}{ No.of tests } & \multicolumn{2}{c}{ Diffusion time $(\mathrm{sec})$} \\
\cline { 3 - 4 } & & Mean & Range \\
\hline Saline & 5 & 36.8 & $34.5-39.5$ \\
Orange juice & 5 & 45.2 & $37.8-47.9$ \\
Milk & 5 & 38.2 & $32.6-43.0$ \\
\hline
\end{tabular}

TABLE 2. Correlation coefficient of each solution.

\begin{tabular}{lcccc}
\hline solution & No.of tests & $\begin{array}{c}\text { Correlation } \\
\text { coefficient }(r)\end{array}$ & Slope & Intercept \\
\hline Saline & 12 & 0.98 & 0.999 & -0.002 \\
Orange juice & 12 & 0.96 & 1.051 & -0.096 \\
Milk & 12 & 0.93 & 0.999 & -0.061 \\
Total & 36 & 0.95 & 1.012 & -0.049 \\
\hline
\end{tabular}




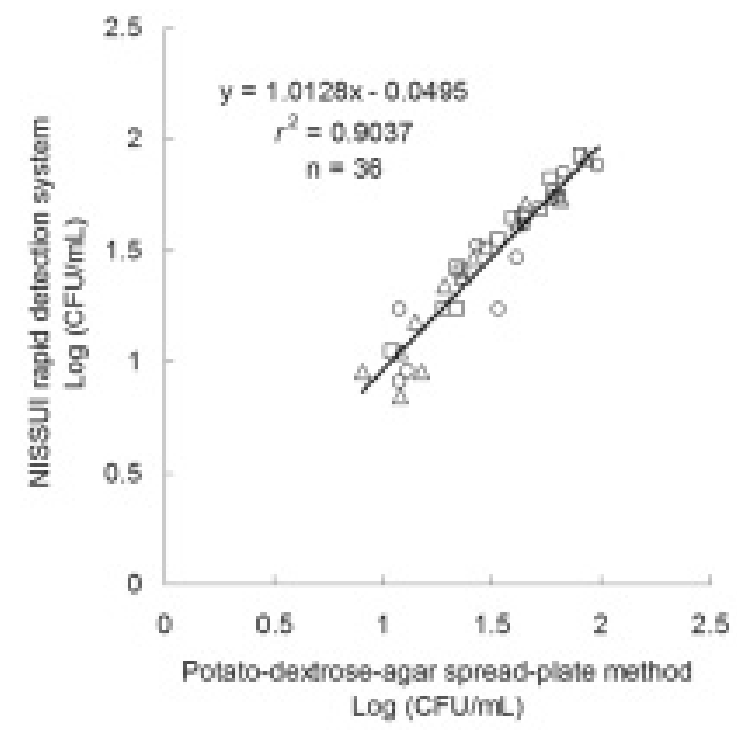

FIG. 2. Correlation between the NISSUI rapid detection system and the conventional potato-dextroseagar spread-plate method. Twelve yeast and mold strains were independently spiked into orange juice, milk, and physiological saline solutions. The numbers of microorganisms inoculated into each sample were determined by the new system after $48 \mathrm{~h}$ incubation and by the potato-dextrose-agar spread-plate method after $120 \mathrm{~h}$ incubation. Symbols: triangle, orange juice; circle, milk; square, physiological saline solution.

molds by the conventional spread-plate method requires proficiency because various colonies show different morphologies, sizes, and colors. This system described herein is an automated microorganismcounting system; therefore, technical expertise is not critical. These features allow for benefits, including a short analysis time for the detection of contaminating yeasts and molds in food.

In this study, we have demonstrated that our novel rapid microbe detection system, the NISSUI rapid detection system, can rapidly detect yeasts and molds in food. Recently, a number of studies have attempted the rapid detection of foodborne microorganisms (Sakakibara et al., 2003, Shimakita et al., 2006, Takahashi et al., 2005). Among these protocols, membrane-filtration method is one of the integral steps. Orange juice contains pulp, which makes the use of membrane filters difficult. It is also difficult to test milk by the membrane-filtration method. Our system enables the detection of yeasts and molds in food without any complex pretreatment.

This is the first report describing the possible advantages of this system. To further evaluate the prospective use of the NISSUI rapid detection system, comprehensive collaborative studies must be performed.

\section{ACKNOWLEDGEMENTS}

The authors thank Mr. Hideaki Shibata and Mr. Yutaka Naka for their helpful suggestions.

\section{REFERENCES}

London, R., Schwedock, J., Sage, A., Valley, H., Meadows, J., Waddington, M., and Straus, D. (2010) An automated system for rapid non-destructive enumeration of growing microbes. PLoS One., 5, e8609.

Renard, A., Gómez di Marco, P., Egea-Cortines, M., and Weiss, J. (2008) Application of whole genome amplification and quantitative PCR for detection and quantification of spoilage yeasts in orange juice. Int. J. Food Microbiol., 126, 195-201.

Sakakibara, T., Murakami, S., and Imai, K (2003) Enumeration of bacterial cell numbers by amplified firefly bioluminescence without cultivation. Anal. Biochem., 312, 48-56.

Shimakita, T., Tashiro, Y., Katsuya, A., Saito, M., and Matsuoka, H. (2006) Rapid separation and counting of viable microbial cells in food by nonculture method with bioplorer, a focusing-free microscopic apparatus with a novel cell separation unit. J. Food Prot., 69, 170-176.

Takahashi, T., Nakakita, Y., Watari, J., and Shinotsuka, K. (2000) Application of a bioluminescence method for the beer industry: sensitivity of MicroStar-RMDS for detecting beer-spoilage bacteria. Rapid Microbe Detection System. Biosci. Biotechnol. Biochem., 64, 1032-1037.

Tournas, V. H., and Katsoudas, E. (2005) Mould and yeast flora in fresh berries, grapes and citrus fruits. Int. J. Food Microbiol., 105, 11-17.

Tournas, V. H., Heeres, J., and Burgess, L. (2006) Moulds and yeasts in fruit salads and fruit juices. Food Microbiol., 23, 684-688.

Wyatt, M. K., Parish, M. E., Widmer, W. W., and Kimbrough, J. (1995) Characterization of mould growth in orange juice. Food Microbiol., 12, 347-355. 